\title{
The Difference in Emoji Usage Between Genders
}

\author{
Yanty Wirza, Della Hanifah Nurbaeti, Hana Hanifah, Hasri Hanifah \\ English Language Education Department \\ Universitas Pendidikan Indonesia \\ Bandung, Indonesia \\ yantywirza@upi.edu
}

\begin{abstract}
This study investigated the use of emoji between male and female genders. Using mixed-method research, the study collected messages with emojis from 20 male and 20 female college students in one of the cities in Indonesia. The study also used brief interviews to learn the reasons and factors for using emojis. The findings show that while there are no signs in terms of intensity of the emoji use, there are some differences in the use of emojis between males and females especially in the kinds of emoji selections. Both genders reported that they used emojis to have to strengthen the meaning of their messages. Besides, the use of emoji is considered to not gender-specific because it functioned to create a comfortable atmosphere in online communication devices for both genders.
\end{abstract}

Keywords: emoji, gender, online communication

\section{INTRODUCTION}

Emoji is one of the mediums that people use in their communication devices to convey their feelings. It is a variety of small images, symbols, or icons used in text fields in electronic communication (Merriam-Webster, n.d.). Text messages and pictures are considered a typical traditional representation of information but emoji are taken as more communicative, realistic and rich in meaning so more preferred by smart-phone users. Emoji was invented by Shigetaka Kurita in 1999 for a Japanese internet platform. At that time, there were only 176 emojis invented. Since then, emoji have gained astonishing popularity and interwoven in our daily communication. The usage of emoji has become a trend among the millennials that even Oxford Dictionary chose "tears of joy" emoji as the word of the year in 2015. As in March 2019, there are a total of 3.019 emojis in the Unicode Standard that people can choose to express their thoughts and ideas.

Each individual is unique in terms of their behavior and their way of thinking. There might be some tendencies in the usage of emojis, especially between genders. Some researches show that women are more often to use emoji rather than men (Chen, $\mathrm{Lu}, \mathrm{Ai}, \mathrm{Li}, \mathrm{Mei}, \& \mathrm{Liu}, 2018$ ). It is also added that compared to men, women tend to use more than one emoji. These interesting findings lead us to question how men and women are different in using emoji. Therefore, we would like to conduct mini-research to find in what extend the differences between men and women in using emoji and what are the factors causing the difference of emoji usage between genders. The study is conducted to investigate whether there are differences between men and women in using emoji and what factors cause the difference of emoji usage between men and women. Men and women have their special characteristics physically and mentally that could not be found within the opposite sex. It means that the communication style of men and women might be specified by gender. The interest in this research is not a new thing as it has been rising since 1990 (Cameron, 2007). One of the famous literature about gender difference might be a book from John Gray which is "Men are from Mars, Women are from Venus", where he stated the problems of communication is caused by fundamental psychological difference. He analogized the characteristic differences in a metaphor that men and women came to earth from their respective distinct planet.

There are many ways to communicate with each other. It divided into two major ways, verbally and nonverbally. Verbal communication is spoken communication, including the use of words and intonation to convey meaning (Levine \& Adelman, 1993). If we do verbal communication, we have to pay attention to our intonation, direct and indirect communication and obey conversation rules; greeting/ opening, discussion of the topic, and closing/ farewell. Meanwhile, non-verbal communication is 'silent' communication, including the use of gestures, facial expressions, eye contact, and conversational distance (Levine \& Adelman, 1993). Non-verbal communications rely on our facial expressions, eye contact, and gestures. Non- verbal has more power than verbal communication because it can express much meaning.

Nowadays, communication extends to a more sophisticated way. There are many platforms that we can use to communicate or chat with people that far away, such as Whatsapp, WeChat, BBM, etc. Those platforms can be called as computer-mediated communication (CMC). CMC is a term that is used to name many forms of human communication through networked computers "which can be synchronous or asynchronous and involve one-to-one, one-to-many, or manyto-many exchanges of text, audio, and/or video messages" (Lee \& Oh, 2017). It could make communication easier 
because the delivery of messages becomes faster and can save time. On the other hand, it's also challenging to convey nonverbal messages through CMC. As stated before, Non-verbal communication relies on facial expressions, eye contact, and gestures, meanwhile, we cannot see how others react to our messages. As it is not readily communicated, it might reduce the quality of social interactions and leads to misunderstanding (Culnan \& Markus1987).

To overcome this, communication through CMC provides a variety of emojis that can be used to convey non-verbal expressions or the feelings of users. Emoji is a small digital image or icon used in electronic messages to express an idea, emotion, etc. People began using emoticons to express affection and to avoid misunderstandings or negative feelings (Huang, et.al, 2008). For the first time, emojis were introduced by Apple among global users in 2011, when Apple Inc. incorporated 722 emojis into the iPhone keyboard. Since then, the number and types of emojis available have rapidly expanded (Lee \& Oh, 2017). This is in line with what Kaye, et. al. (2016) have said that the use of emojis increases when individuals have a conversation through online media, for example, using instant messaging apps to communicating.

Currently, emoji have been considered as a tool to convey thoughts and feelings as nonverbal signs in speech (Crystal, 2006; Rezabek \& Cochenour, 1998; Wolf, 2000). Emoji has a similar function as a human language (Pavalanathan \& Eisenstein, 2015). It can represent gestures or facial expressions that commonly used in non-verbal language. Moreover, emoji can make a digital communication livelier, because it could elucidate the meaning of plain text messages (Hakami, 2017). If a text message uses only words without punctuation or emojis, people might have various arguments whether the message contains a negative or positive meaning. If the message is added an emoji, then it can lead to a livelier message and we can find out the sender's purpose in sending that, whether the meaning or feeling is negative or positive. Yang (2018) classify emojis as "(a) devices of modesty; (b) hedging devices; (c) to soften requests; (d) positive politeness strategies; (e) in rapport building; (f) humor; (g) to help convey emotion; (h) emphatic use; and (i) lexical use".

Dresner \& Herring (2010) argued that emoticons indicate the speaker's intention, and they concluded three linguistic functions for emoticons. "First, they consider emoticons as emotional indicators that are directly correlated to facial expressions, e.g. sad or angry. Second, they consider emoticons as non-emotional indicators that are also correlated to facial expressions, e.g. joking. Last, they consider emoticons as illocutionary force indicators that are not correlated to facial expressions but deep intention". When people feel happy, they will give a positive emoji such as smile, laugh out loud emoji, or heart-shaped eyes. On the contrary, people tend to use negative emoji when they feel down such as crying, the red face that indicates anger, or a pensive face. Emoji are also used for some specific topics, such as in sexually suggestive contexts (Thomson, Kluftinger, \& Wentland, 2018). It may be useful tools in creating a relationship between partners. The face with tears of joys has been nominated by Oxford University Press in 2015 as the word of the year. Since then, the Oxford University Press has partnered with the SwiftKey company, the author of the most recent detailed analysis of emoji around the world (SwiftKey, 2015).

In communicating, people tend to use verbal communication and also non - verbal communication, some of non - verbal communicating are facial expressions and body language. These include the part of our daily communication to enhance the effects on our sentiments and emotions. In nowadays, technology is up to date, people also can communicate through media, for example, the chatting platforms. In $93 \%$ of everyday communication, people use a symbolic representation of their messages and emotions. Emoji usually use to describe or show their feelings, expression, and sentiments. Emoji is a set of pictographic Unicode characters that use to express humor, irony, and sarcasm and intimacy. Emoji is not something that we hardly can find. It is usually found in any platforms, it can be found in a comment section, in a chat, in someone's caption or description, and many more. Several people might think that the use of emoji tends to be a habit. For several people, the use of emoji might consider as an important thing, which they would not get satisfied if they don't use emoji, and the rest might think the opposite. Emoji does not only function on the semantic level, however, and the reasons for their use do not derive solely from their semantics. Emoji usually describe as something important, it also has meaning; thus it is also important to consider their pragmatic functions in social media discourse. Well, I found several studies that claim that women tend to use emoji more than men. Emoji also have some limited variation of symbols, non-standard formation, and their use bring in some questions to data analysis whereas emoji has standard creation. They consider not only to express feelings but also consider to be able to represent other objects as well.

Tossell et al. (2012) stated that emoticons or emoji bring big effects, in text messaging and in particular, how much gender differences exist in the frequency and variety of emoticons used. According to Nancy Briton and Judith Hall, when it comes to sending, receiving, and interpreting nonverbal cues during the conversation, women are better than men. It is established, speech females tend to use more cues than men, in one day. The study also shows that the nonverbal behaviors used, including using an emoji tend to be used by women because they try to be more expressive through it. Females are also more likely to use emoji that they might think as expressive in real-time written communication such as chatting or texting.

Emoji were originally designed and made with the purpose to help express sentiments or describe the expression compactly and vividly. Recent research also demonstrates that expressing sentiment and showing expression is the main intention of using emoji. We infer that the gendered patterns of using emoji (i.e., frequency and preference) can be implicitly affected by the way sentiment is expressed. For example, it is widely believed that women are more emotional and more expressive than men. The study shows that the percentage of the female is bigger than male, and it can be 
concluded that consistent with the existing belief, women are more emotional than men.

Nishimura (2015) also stated that from 200 participants in a Japanese blog site established in 2004, having 121 major categories and some 7000 sub-categories. He found that women are more inclined to send emoji compared to men. $\mathrm{Lu}$ et. al. (2016) found that study the data set consisting of 400 emoji containing texts of 3.87 million active users from 212 countries and the sender or users could be known or predicted through the emoji usage. Chen et.al (2018) found the findings that the gender-based use of emoji, for these purposes there were 134419 active participants from 183 countries were selected and 401 million texts were saved in 90 days. The findings show that there is a difference in using emoji between both genders. Similarly, however, we could not find any study with the largest sample about emoji usage from gender perspectives. Wolf (2000) explored emoji observation among different gender groups and concluded that there were significant differences among the same and different gender groups.

\section{METHOD}

This type of research was a case study. The research was chosen because this method might be in the form of qualitative or quantitative to analyze the data. Moreover, this research is one of the cases in analyzing a person's behavior. The researchers in this study focused on the differences in emoji usage by men and women and their reason in using emoji. The step in collecting data have distributed the questionnaire, interview, interpretation of the interview, and then analyze the result. The questionnaire was given to know respondents' opinions about using emoji and determine the participant to be interviewed. The interview was conducted to gain more information from participants. The participants were active college students in one of the cities in Indonesia, consisting of 20 female students and 20 male students.

The data of this study were in the form of qualitative data. Qualitative data were used to clarify the quantitative data, enhancing the trustworthiness of the data, and the subjectivity in analyzing the data. A process usually called triangulation (Dornyei, 2007). The researchers got the data from many resources. It aimed to gather multiple perspectives on the situation being studied.

\section{FINDINGS AND DISCUSSION}

The research finding is presented to answer the research questions that previously mentioned in the first chapter. The data were collected by using triangulation techniques by using questionnaires and interviews to find a richer and deeper understanding of the issue. In this section, we will present the difference of emoji usage between genders. We classified the data into three units, there are a. the intensity of the emoji usage and $b$. the difference of emoji usage between genders.

\section{A. The intensity of the emoji usage between men and women}

To find out the intensity of emoji usage, we utilize SPSS to find out the different intensity between genders.
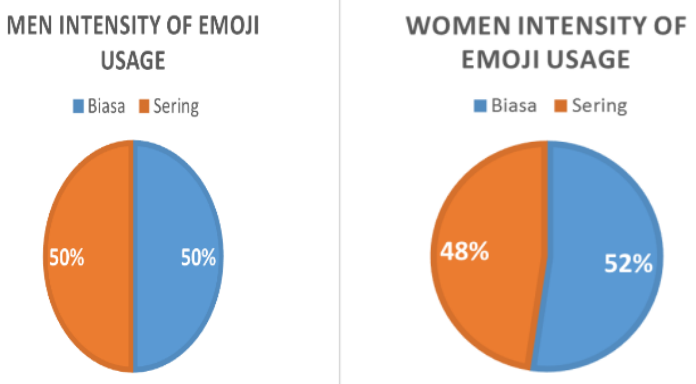

Fig. 1. MEN AND WOMEN INTENSITY OF EMOJI USAGE

The participants consist of 20 male participants and 20 female participants. Based on Figure 1. above, it can be seen that there is a different scale between men and women. The pie chart on the left, which explained men's intensity of emoji usage, showed that half of them were often using emoji, while the other half is not so much. While for women participant, 11 participants were not often using emoji, while only nine of them that were often using emoji. Therefore, each percentage in the pie chart on the left showed $52 \%$ and $48 \%$.

To find out the intensity difference between men and women, the first thing to do is to find out the normality of the data.

TABLE I. TEST OF NORMALITY

Tests of Normality

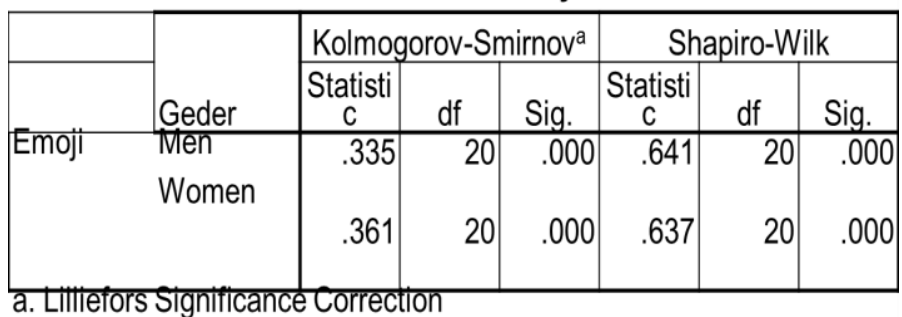

Based on Table I. above, data distribution is not normal, so the statistics used are non -parametric statistics. Therefore, the Mann Whitney test is used to analyze 2 independent data as shown in Table II.

TABLE II. RANKS

\begin{tabular}{|r|l|r|r|r|}
\hline \multicolumn{1}{|c|}{} & & & & \\
\hline Emoji & Gender & $\mathrm{N}$ & Mean Rank & Sum of Ranks \\
\hline & Men & 20 & 21.00 & 420.00 \\
& Women & 20 & 20.00 & 400.00 \\
& Total & 40 & & \\
\hline
\end{tabular}


TABLE III. TEST STATISTICS

\begin{tabular}{|l|r|}
\hline \multicolumn{2}{|c|}{ Test Statistics $^{\mathbf{a}}$} \\
\hline \multicolumn{2}{|c|}{ Emoji } \\
\hline Mann-Whitney U & 190.000 \\
Wilcoxon W & 400.000 \\
$Z$ & -.313 \\
Asymp. Sig. (2-tailed) & .755 \\
Exact Sig. [2*(1-tailed Sig.)] & $.799^{\mathrm{b}}$ \\
\hline a. Grouping Variable: Gender \\
b. Not corrected for ties.
\end{tabular}

Based on the tables III. showed the data above, the p-value showed by Asymp. Sig (2-tailed) $>\alpha$ with $(\alpha=0,05)$. So we can conclude that there is no significant difference intensity in using emoji between males and females.

2. The difference of emoji usage between men and

women

\section{a. The Choice of Emojis}

From the questionnaire that we gave to respondents, we have found significant differences between females and males in using emojis. It found out that the most frequently used emojis by men and women is (face with tears of joy) which some of them considered this emoji to be the most suited emoji that could express their feeling. We also found the other differences for particular emojis choice by women and men. In our finding, (loudly crying face) is the second most popular emoji that women usually use, while (folded hand) emoji is the second most used emoji by men.

\section{B. The Function of Emoji Usage}

Related to Kavanagh's theory (2010), (face with tears of joy) emoji is considered to express some of the functions of the emoticon, which are humor function and humanize online interaction function. It can be seen that this emoji is considered to be a gender-neutral emoji because not only it is the most used emoji between the genders, but also how it functioned to create a comfortable atmosphere in online communication devices.

Based on our research findings, our data showed similarities with Chen et al (2018), that the most used emoji by women and men is (face with tears of joy) emoji, as it is considered to be useful and practical to express their feelings. Related to Kavanagh's theory (2010), (face with tears of joy) emoji is considered to express some of the functions of the emoticon, which are humor function and humanize online interaction function. It can be seen that this emoji is considered to be a gender neutral emoji because not only it is the most used emoji between the genders, but also how it functioned to create a comfortable atmosphere in online communication devices.

\section{CONCLUSIONS}

Based on our research, we can conclude that there is no significant difference of the intensity and the function in emoji usage between men and women. However, there is a difference in choosing emoji, and also the amount of emoji used between genders to communicate intensity of meanings and feelings contained in the message. This study was conducted with limitation; in which it is mainly a preliminary research that recruited a small number of participants. As for the recommendation, studies of the use of emojies are potentially useful for linguistics research. Despite the emoji usage was different between men and women; it is worth to remember that these differences do not affect the function of emoji, which is to convey ideas. Therefore, emoji should be considered to be as important as other linguistic features, as the use of emojis might be a reliable indicator for users in different languages.

\section{REFERENCES}

Briton, N. J., \& Hall, J. A. (1995). Beliefs about female and male nonverbal communication. Sex Roles, 32(1-2), 79-90. doi: 10.1007/bf01544758

Cameron, D. (2007). Do men and women share the same language? [Online]. Retrieved 2019, from https://www.theguardian.com/world/2007/oct/01/gender.books

Chen, Z., Lu, X., Ai, W., Li, H., Mei, Q., \& Liu, X. (2018). Through a gender lens: Learning usage patterns of emojis from large-scale android users. WWW 2018: The 2018 Web Conference April 23-27, 2018, Lyon, France (p. 10). New York: ACM.

Crystal, D. (2006). Language and the internet. Cambridge, UK: Cambridge University Press.

Culnan M, Markus ML. (1987). Information technologies. In: Jablin FM, Putnam LL, Roberts KH, Porter LW, editors. Handbook of organizational communication: An interdisciplinary perspective. Newbury Park, CA: Sage; 1987. pp. 420e444.

Dornyei, Z. (2007). Research methods in applied linguistics. New York: Oxford University Press.

Gray, J. (1992). Men are from Mars, women are from Venus. New York: Harper Collins

Cameron, D. (2007). Do men and women share the same language? [Online]. $\quad$ Retrieved 2019, from https://www.theguardian.com/world/2007/oct/01/gender.books

Emoji. (n.d). In Merriam-Webster.com. [Online]. Retrieved 2019, from https://www.merriam-webster.com/dictionary/emoji

Gray, J. (1992). Men are from mars, Women are from venus. New York: Harper Collins.

Hakami, S. A. (2017). The importance of understanding emoji: An investigative study. Thesis. School of Computer Science. University of Birmingham

Huang AH, Yen DC, Zhang X. (2008). Exploring the potential effects of emoticons. Information \& Management 2008, 45, 466-473. doi: 10.1016/j.im.2008.07.001

Kaye, L. K., Wall, H. J., \& Malone, S. A. (2016). Turn that frown upsidedown: A contextual account of emoticon usage on different virtual platforms. Computers in Human Behavior, 60, 463-467 doi: 10.1016/j.chb.2016.02.088

Kavanagh, B. (2010). A cross-cultural analysis of Japanese and English nonverbal online communication: The use of emoticons in weblogs. Intercultural Communication Studies, 3, 65-80. doi: 10.1016/j.langcom.2016.03.003

Lee, E. J., Oh, S. (2017). Computer-mediated communication. Oxford Biblioghraphies

Levine, D. R., Adelman, M. B. (1993). Beyond language: Cross-cultural communication.Englewood Cliffs, N.J: Prentice-Hall.

Nishimura, Y. (2015). A sociolinguistic analysis of emoticon usage in Japanese blogs: Variation by age, gender, and topic. AoIR Selected Papers of Internet Research, 5. 
Oxford Dictionaries. (2015). Announcing the Oxford Dictionary "Word" of the year 2015. Retrieved from https://languages.oup.com/press/news/2019/7/5/WOTY

Pavalanathan, U., \& Eisenstein, J. (2015). Emoticons vs emojis on Twitter: a causal inference approach. [Online]. Retrieved from http://www.arXiv.org/abs/1510.08480.

Rezabek, L. L., \& Cochenour, J. J. (1998). Visual cues in computer-mediated communication: supplementing text with emoticons. Journal of Visual Literacy, 18(2), 201-215. doi: 10.1080/23796529.1998.11674539

SwiftKey. (2015). Swiftkey emoji report, April 2015. [Online]. Retrieved from https://goo.gl/9QXoEn. Unicode® Technical Report \#51

Thomson, S., Kluftinger, E., and Wentland, J. (2018). Are you fluent in sexual emoji?: Exploring the use of emoji in romantic and sexual contexts. The Canadian Journal Human Sexuality. 27, 226-234. doi: 10.3138/cjhs.2018-0020

Tossell, C. C., Kortum, P., Shepard, C., Barg-Walkow, L. H., Rahmati, A., \& Zhong, L. (2012). A longitudinal study of emoticon use in text messaging from smartphones. Computers in Human Behaviour, 28(2), 659-663.

Wolf, A. (2000). Emotional expression online: Gender differences in emoticon use. CyberPsychology \& Behavior, 3(5), 827-833.

Yang, Y. (2018). Pragmatic functions of emoji in internet-based communication: A corpus-based study. Asian-Pacific Journal of Second and Foreign Language Education December 2018, 316 